\title{
The Use of Data Collection and Big Data Analysis in Neurodegenerative Disease Prevention
}

\author{
Georgios Nikiforakis
}

\section{Abstract}

Data collection and analysis is becoming more and more important in our everyday lives. It is widely believed that with the power of data collection, almost every problem can be given a possible solution. Machine learning and artificial intelligence algorithms along with the use of the Alzheimer's Disease Neuroimaging Initiative (ADNI) database that collects data from subjects suffering from Alzheimer's disease (AD) can be utilised for recognising biomarkers' patterns that suggest degenerative behavior. Thus, allowing for early prevention. Computers are able to distinguish patterns over millions of data faster than humans can.
The suggested methodology will be able to precisely find subjects that might suffer from such diseases due to certain patterns and biomarkers that suggest such predisposition at an early and thus treatable stage of the disease. Due to the quantity of the data collected, an algorithm will be able to distinguish even the slightest change in a healthy neuron in order to treat it. Other databases can be used for other diseases without the concept being changed.

\section{Keywords}

Machine learning - Artificial intelligence . Biomarkers $\cdot$ Pattern recognition 